\title{
Sex significantly influences transduction of murine liver by recombinant adeno-associated viral vectors through an androgen-dependent pathway
}

\author{
Andrew M. Davidoff, Catherine Y. C. Ng, Junfang Zhou, Yunyu Spence, and Amit C. Nathwani
}

\begin{abstract}
A systematic evaluation of the influence of sex on transduction by recombinant adeno-associated viral vector (rAAV) indicated that transgene expression after liver-targeted delivery of vector particles was between 5- to 13-fold higher in male mice compared with female mice, irrespective of the proviral promoter or CDNA and mouse strain. Molecular analysis revealed that the rAAV genome was stably retained in male liver at levels that were 7-fold higher than those observed in females. Further, the sex difference in transduction was observed with AAV-2- and AAV-5-based vectors, which use distinct receptor complexes for infection. In con-
\end{abstract}

cordance with the differences in AAV transduction, gel shift analysis with nuclear extracts derived from the liver of mice and humans revealed substantially higher binding of host nuclear protein to the rep-binding site (RBS) of AAV inverted terminal repeat (ITR) in males compared with females. Transduction efficiency and binding of nuclear protein to RBS was dramatically reduced in male mice by castration. In contrast, although oophorectomy did not significantly influence rAAV transduction, administration of $5 \alpha$ dihydrotestosterone, prior to gene transfer, increased stable hepatocyte gene transfer in females to levels observed in male mice, implying that androgens significantly influence hepatocyte gene transfer. Interestingly, sex did not have a significant effect on AAV gene transfer into nonhepatic tissue, indicating that there are distinct tissue- and sex-specific differences in the mechanisms responsible for efficient transduction with this vector. These results have significant implications for gene therapy of autosomal and acquired disorders affecting the liver. (Blood. 2003;102:480-488)

두 2003 by The American Society of Hematology

\section{Introduction}

Recombinant adeno-associated viral (rAAV) vectors are proving to be highly effective vehicles for the gene therapy of disorders that affect the liver such as hemophilia, $\alpha 1$ antitrypsin deficiency, and mucopolysaccharidosis. ${ }^{1-3}$ The success of these vectors can be ascribed to the fact that they are able to mediate persistent therapeutic expression of transgene such as coagulation factor IX (FIX) in murine and canine models of hemophilia B after portal vein administration of vector particles. ${ }^{4-12}$ Recently, we have demonstrated sustained expression ( $>18$ months) of human FIX (hFIX) at $4 \%$ to $10 \%$ of physiologic levels in a nonhuman primate model following liver-targeted delivery of AAV vector genomes. ${ }^{1}$ Other advantages afforded by AAV vectors include the best safety profile among gene transfer vectors of viral origin as wild-type AAV has never been associated with any human disease. Safety is further enhanced by the dependence of AAV on coinfection with helper virus (usually adeno or herpes virus) for productive infection. Additionally, recombinant vectors are entirely devoid of wild-type viral genes, thus reducing the potential for invoking cell-mediated immune response to foreign viral proteins. ${ }^{13}$ These attributes have led to a phase 1 to 2 study to evaluate the safety of rAAV vectors for the treatment of hemophilia B. ${ }^{14,15}$

Major obstacles to the successful transition of AAV vectors to the clinic include the enormous viral titers required for efficient transduction and problems inherent in scale up of production to generate sufficient vector particles for gene transfer in adults. New insights into the biology of $\mathrm{AAV}$ vectors, however, offer an opportunity to enhance the potency of AAV, thereby reducing the ultimate titers required for clinical application. The identification of heparan sulfate proteoglycan (HPG) ${ }^{16}$ as the primary receptor for AAV-2-based vectors has led to the utilization of heparin affinity chromatography to generate rAAV particles with significantly improved biologic activity over vector preparations purified by cesium chloride gradient centrifugation. ${ }^{17,18}$ Following internalization, the rAAV virions are routed to the late endosomal compartment prior to release in the perinuclear region, a process that is associated with considerable degradation of viral particles. ${ }^{19,20}$ Agents that modulate the ubiquitin-proteasome system augment nuclear trafficking of viral particles with a concurrent increase in AAV transgene expression. ${ }^{19-21}$ Following translocation of the AAV particles into the nucleus, stable gene transfer efficiency is further limited by the need to convert the single-stranded viral genome into a double-stranded transcriptionally active form by a mechanism that remains obscure. ${ }^{22,23}$ Adenoviral E2b and E4orf6 proteins and a variety of genotoxic agents, however, facilitate this process, leading to higher gene transfer efficiency. ${ }^{19,22-24}$ In the absence of helper virus function, cellular factors, including the single-stranded
From the Department of Surgery, St Jude Children's Research Hospital, Memphis, TN; Division of Experimental Hematology, St Jude Children's Research Hospital, Memphis, TN; Department of Haematology, University College London, United Kingdom; and National Blood Authority, London, United Kingdom.

Submitted September 23, 2002; accepted February 24, 2003. Prepublished online as Blood First Edition Paper, March 13, 2003; DOI 10.1182/blood-2002-09-2889.

Supported by the Assisi Foundation of Memphis and the American Lebanese Syrian Associated Charities (ALSAC) and by a grant from the Katharine
Dormandy Trust, United Kingdom (A.C.N.).

Reprints: Andrew M. Davidoff, Department of Surgery, St Jude Children's Research Hospital, 332 N Lauderdale, Memphis, TN 38105; e-mail: andrew.davidoff@stjude.org.

The publication costs of this article were defrayed in part by page charge payment. Therefore, and solely to indicate this fact, this article is hereby marked "advertisement" in accordance with 18 U.S.C. section 1734

(C) 2003 by The American Society of Hematology 
D sequence binding tyrosine phosphoprotein (ssD-BP), are believed to directly mediate second strand synthesis and their effects can be enhanced by inhibitors of tyrosine kinase. ${ }^{25}$

The AAV provirus is stably maintained in only $5 \%$ of hepatocytes ${ }^{26}$ a transduction efficiency that is considerably lower than that observed with other postmitotic tissues such as brain, ${ }^{27}$ retina, ${ }^{28}$ and skeletal muscle. ${ }^{5,6,14,26,29}$ The reasons for the tissuespecific differences in transduction also remain a mystery. Additionally, episomal forms of rAAV that persist for periods exceeding 18 months are primarily responsible for transgene expression after liver-targeted delivery of vector particles as integration of the provirus occurs in less than $10 \%$ of transduced cells. ${ }^{1,30}$ This situation raises concerns that expression may be lost with hepatocyte turnover, particularly as the mechanisms responsible for the persistence of the extrachromosomal AAV genome are poorly understood. In this study we describe a novel androgen-dependent pathway for augmenting hepatocyte gene transfer that leads to 7 -fold higher stable transduction of the liver in male mice compared with female mice. Moreover, treatment of female mice with testosterone prior to infection by rAAV augments transduction of the liver to levels observed in males. These observations provide new insights into the biology of rAAV vectors and have significant implications for the optimal use of this vector for the treatment of autosomal and acquired disorders affecting the liver.

\section{Materials and methods}

\section{rAAV vector construction and purification}

The AAV-2-based vectors, rAAV-2 CAGG-FIX and rAAV-2 CAGGtsFlk-1, are shown diagrammatically in Figures $1 \mathrm{~A}-2 \mathrm{~A}$, respectively, and have been described before. ${ }^{4,31}$ rAAV-2 LSP-FIX vector that contains the $\alpha 1$-microglobulin/bikunin enhancer and thyroid hormone-binding globulin promoter (referred to as liver-specific promoter, LSP) is identical to the vector described by Wang et al, ${ }^{9}$ except that it contains the human FIX cDNA instead of the canine cognate (Figure 2B). rAAV-5 CAGG FIX was generated after subcloning the CAGG-FIX expression cassette into the $B g l I I$ and SalI sites of plasmid pAAV5-7D05 (a gift from Dr Robert Kotin, National Institutes of Health [NIH], Bethesda, MD) containing type 5 inverted terminal repeats (ITRs). ${ }^{32}$ The AAV-2 vectors were made by the transient transfection method described before and purified by heparin affinity column chromatography. ${ }^{4} \mathrm{rAAV}-5$ CAGG-FIX vector particles were produced in an identical fashion, except packaging plasmid pAAV5-2 (a gift from Dr Robert Kotin, NIH) was used instead of XX2. The AAV-5 virions were purified using mucin affinity column chromatography. ${ }^{33}$ The vector stocks were consistently free of contamination with wild-type (wt) AAV and cellular and adenoviral proteins as judged by methods described previously. ${ }^{4,18}$

\section{In vivo transduction and animal procedures}

All animals were treated according to the standards set in the Guide for Care and Use of Laboratory Animals (NIH publication no. 85-23). All procedures were performed in accordance with guidelines under protocols approved by the Institutional Animal Care and Use Committee at St Jude Children's Research Hospital (Memphis, TN). B6.CB17-Prkdc-SzJ immunodeficient mice were obtained from the Jackson Laboratory (Bar Harbor, ME), whereas C.B-17 severe combined immunodeficient (SCID) mice together with $\mathrm{C} 57 \mathrm{~B} 1 / 6$ and $\mathrm{A} / \mathrm{J}$ immunocompetent mice were obtained from Charles River Laboratory. Portal vein, tail vein, and intramuscular administration of rAAV vector particles was performed in 7- to 10-week-old mice as described before. ${ }^{4}$ Bilateral oophorectomies involved a lower midline incision to access the peritoneal cavity. The ovaries were identified adjacent to each fallopian tube and removed. The midline incision was then closed with a continuous full-thickness absorbable suture. $5 \alpha$ dihydrotestosterone (DHT; $0.05 \mathrm{mg}$ ) in the form of slow release pellets (21-day release profile;
Innovative Research of America, Sarasota, FL) were implanted into the subcutaneous tissue through a small incision in the dorsal skin. To evaluate the influence of sex hormones on transduction efficiency, $1 \times 10^{11} \mathrm{rAAV}-2$ CAGG-FIX vector particles were injected into the tail vein of cohorts of female mice a month before or after bilateral oophorectomy. In separate experiments, DHT was implanted in male or oophorectomized female mice 2 weeks prior to tail vein administration of $1 \times 10^{11}$ rAAV CAGG-FIX vector particles. To further assesses the effect of androgens, 3-month-old male $\mathrm{C} 57 \mathrm{Bl} / 6$ mice were castrated using a transabdominal approach after anesthesia with $2 \%$ isoflurane. rAAV-2 CAGG-FIX vector particles $\left(1 \times 10^{11}\right)$ were injected into the tail vein of these mice after 2 weeks together with concurrent control cohorts consisting of age-matched unmanipulated male and female mice. Blood samples were collected at regular intervals from the retro-orbital plexus and assayed for the appropriate transgene (hFIX or tsFlk-1) by the enzyme-linked immunosorbent assay (ELISA) methods described previously. ${ }^{4,31}$ The probability of statistical difference between experimental groups was determined by one-way analysis of variance (ANOVA) and paired Student $t$ test using GraphPad Prizm version 3.0 software (GraphPad, San Diego, CA).

\section{Nucleic acid isolation and analysis}

Low-molecular-weight Hirt DNA was extracted from male and female liver at day 3, 10, and 20 after liver-targeted delivery of $1 \times 10^{11}$ rAAV-2 CAGG-FIX vector particles using a modification of a previously described method. ${ }^{34}$ In brief, 100 to $200 \mathrm{mg}$ liver tissue was homogenized in $6 \mathrm{~mL}$ lysis buffer ( $400 \mathrm{mM} \mathrm{NaCl}, 10 \mathrm{mM}$ Tris (tris(hydroxymethyl)aminomethane), $\mathrm{pH} 7.5,10 \mathrm{mM}$ EDTA (ethylenediaminetetraacetic acid) using a Dounce homogenizer. Cells were disrupted by adding $400 \mu \mathrm{L} 10 \%$ sodium dodecyl sulfate (SDS). Contaminating RNA was degraded by adding $2 \mu \mathrm{L}$ of $10 \mathrm{mg} / \mathrm{mL}$ DNase-free RNase I and incubating the solution for 1 hour at $37^{\circ} \mathrm{C}$. Then, $1 \mathrm{~mL}$ of $5 \mathrm{M} \mathrm{NaCl}$ was added to the suspension and incubated overnight at $4^{\circ} \mathrm{C}$. Cellular debris was removed by centrifugation at $17000 \mathrm{~g}$ for 30 minutes at $4^{\circ} \mathrm{C}$. The DNA in the supernatant was ethanolprecipitated, and the pellet was resuspended in nuclease-free $\mathrm{H}_{2} \mathrm{O}$. Undigested Hirt DNA $(10 \mu \mathrm{g})$ was separated on $1 \%$ agarose gel, transferred to nitrocellulose by Southern blotting, and hybridized with a $\alpha^{32} \mathrm{P}$-labeled 1.6-kb hFIX cDNA probe at $42^{\circ} \mathrm{C}$. The intensity of the hybridization was determined using the STORM phosphorimager and ImageQuant software (Molecular Dynamics, Sunnyvale, CA).

High-molecular-weight genomic DNA was extracted from liver tissue derived from cohorts of male and female mice 10 weeks after portal vein or tail vein infusion of $1 \times 10^{11} \mathrm{rAAV}-2$ CAGG-FIX vector particles using our previously described method. ${ }^{4}$ DNA was digested with a combination of SpeI and HindIII or NheI alone to release internal fragments of $3.3 \mathrm{~kb}$ and $1.5 \mathrm{~kb}$, respectively, containing the hFIX cDNA. The fragments were size fractionated by electrophoresis through a $0.8 \%$ agarose gel. Prior to transfer onto a nylon membrane (Hybond-N+; Amersham Biosciences, Arlington Heights, IL) by Southern blotting, consistent loading of genomic DNA on the gel was documented using AlphaImager software version 3.24 (Alpha Innotech, San Leandro, CA). The blots were hybridized with a $\alpha^{32} \mathrm{P}$-labeled 1.6-kb hFIX cDNA probe at $42^{\circ} \mathrm{C}$, and the intensity of hybridization was determined as described in "Nucleic acid isolation and analysis." Total cellular RNA was isolated from 20 to $30 \mathrm{mg}$ liver tissue derived from cohorts of male and female mice 10 weeks after transduction with $1 \times 10^{11}$ rAAV-2 CAGG-FIX vector particles using RNA STAT-60 (TEL TEST, Friendswood, TX). Approximately $10 \mu \mathrm{g}$ total RNA from each sample was subjected to denaturing agarose electrophoresis. Prior to transfer onto nylon membrane (Hybond-N+; Amersham Biosciences) by Northern blotting procedure, the integrity of the RNA was assessed by quantitating ribosomal RNA $18 \mathrm{~S}$ and, $28 \mathrm{~S}$, in each sample using AlphaImager software version 3.24 (Alpha Innotech). The blots were hybridized with a $\alpha^{32} \mathrm{P}$-labeled 1.6-kb hFIX cDNA probe at $42^{\circ} \mathrm{C}$, and the intensity of hybridization was determined as described in "Nucleic acid isolation and analysis."

To evaluate the biodistribution of vectors following tail vein administration of rAAV-5 CAGG-FIX, $0.5 \mu \mathrm{g}$ genomic DNA extracted from the liver, spleen, lungs, and kidneys was subjected to polymerase chain reaction (PCR) using FIX primers and conditions described previously for amplification of a 512-bp region of the FIX transgene. ${ }^{1}$ Integrity of DNA was 
determined by amplifying a 604-bp region of the murine $\beta$-actin gene using specific primers and conditions outlined in Nathwani et al. ${ }^{1}$ The relative intensity of the signal was assessed digitally using 1D image analysis software (V3.6) (Kodak, Herts, United Kingdom).

\section{Western blot analysis to detect murine FGFR-1}

Snap-frozen liver tissue $(100-150 \mathrm{mg})$ from naive male and female mice was homogenized in lysis buffer consisting of $25 \mathrm{mM}$ Tris-HCl, $0.5 \%$ NP-40, $0.5 \%$ deoxycholate, $0.2 \%$ SDS, $\mathrm{pH} 8.0$, together with minuteComplete protease cocktail and $1 \mathrm{mg} / \mathrm{mL}$ Pefabloc (Roche Molecular Biochemicals, Indianapolis, IN). Crude cellular supernatant was harvested following centrifugation, and protein concentration was determined using a colorimetric assay (Bio-Rad Laboratories, Hercules, CA). A volume of 40 $\mu \mathrm{g}$ of each sample was separated by $12 \%$ polyacrylamide gel under reducing conditions followed by electrophoretic transfer of proteins to a poly(vinylidene diflouride) (PVDF) membrane. The blots were blocked with $2 \%$ milk and probed with an FGF-Receptor (Ab-1) mouse monoclonal antibody at a concentration of $2.5 \mu \mathrm{g} / \mathrm{mL}$ (Oncogene Research Products, Boston, MA). After washing, the blots were exposed to a horseradish peroxidase (HRP)-conjugated rabbit antimouse immunoglobulin $\mathrm{G}$ (IgG; 1:10 000) secondary antibody included in the electrogenerated ECL chemiluminescence (ECL) kit (Amersham Biosciences), which was used to develop the signal.

\section{Cell culture and in vitro transduction with rAAV vectors}

Human hepatocellular carcinoma $(\mathrm{HuH7})$ cells were chosen for in vitro evaluation of rAAV vectors because these cells express a high level of transgenes following transduction with $\mathrm{rAAV}$, perhaps because they constitutively express adenoviral E1 genes. ${ }^{35}$ These and CHO-K1 (Chinese hamster ovary) cells were maintained in Dulbecco modified Eagle medium supplemented with $10 \%$ fetal calf serum (FCS), $100 \mathrm{U}$ penicillin per $\mathrm{mL}$, and $0.1 \mathrm{mg}$ streptomycin per $\mathrm{mL}(\mathrm{D} 10)$, at $37^{\circ} \mathrm{C}$ in a humidified atmosphere containing $5 \% \mathrm{CO}_{2}$. Five days prior to transduction, the cells were washed and resuspended in serum-free hepatocyte culture media (Clonetic, Walkersville, MD). To assess the influence of androgens on rAAV-mediated gene transfer, $300 \mathrm{nM}$ DHT (Sigma, St Louis, MO) was added to serum-free hepatocyte culture media 5 days prior to gene transfer. HuH7 cells $\left(1 \times 10^{5}\right)$ were transduced overnight in serum-free media with $1 \times 10^{8}$ rAAV-2 CAGG-FIX vector genomes. Medium was conditioned for 24 hours and collected on day 4 after transduction and assayed for hFIX using the Asserachrom IX:ag ELISA kit (American Bioproducts, Parsippany, NJ).

\section{Cy3 labeling of rAAV-2 and analysis in $\mathrm{HuH7}$ cells}

Lyophilized $N$-hydroxysuccinimide (NHS)-ester carbocyanine Cy3 dye was dissolved in conjugation buffer (0.1 M sodium carbonate [pH 9.3]). Heparin affinity-purified rAAV-2 CAGG-FIX particles $\left(2 \times 10^{11}\right)$ were incubated for 30 minutes at room temperature with the $\mathrm{Cy} 3$ dye in a reaction volume of $1 \mathrm{~mL}$. The solutions were then transferred to dialysis cassettes (10 000 molecular weight cutoff; Pierce Biotechnology, Rockford, IL) and dialyzed for 24 hours against 2 changes of buffer containing $20 \mathrm{mM}$ HEPES ( $N$-2-hydroxyethylpiperazine- $N^{\prime}$-2-ethanesulfonic acid) $(\mathrm{pH} 7.5)$ and 150 $\mathrm{mM} \mathrm{NaCl}$. Finally, the vector samples were concentrated in Centricon 30 ultrafilters (Millipore, Bedford MA). Dye-to-virus particle (D/P) ratios of the Cy3-labeled virus samples were calculated according to the manufacturer's instructions (Amersham Biosciences) and were approximately equal to 1 . The labeled $\mathrm{Cy} 3$-virus solution was used directly for infecting naive and DHT-treated $\mathrm{HuH} 7$ cells (multiplicity of infection [MOI] of 1000 DNA particles/cell) on glass slides at $4^{\circ} \mathrm{C}$ for 60 minutes (in the absence of serum). The slides were then washed twice in serum-free Dulbecco modified Eagle medium (DMEM) and fixed immediately prior to analysis. Attachment of Cy3-labeled virus to naive and DHT-treated cells was assessed by confocal microscopy. Nonspecific binding of the labeled rAAV particles was evaluated on $\mathrm{CHO}-\mathrm{K} 1$ cells that are deficient in proteoglycan synthesis.

\section{Preparation of nuclear extracts}

A modification of the method of Dignam et $\mathrm{a}^{36}$ was used to prepare nuclear extract from fresh liver tissue derived from male and female B6.CB17-PrkdcSzJ immunodeficient and C57B1/6 mice. In brief, approximately $10 \mathrm{mg}$ liver tissue was minced, homogenized, and resuspended in $1 \mathrm{~mL}$ hypotonic buffer $(20 \mathrm{mM}$ Tris- $\mathrm{HCl}, \mathrm{pH}$ 7.5, $10 \mathrm{mM} \mathrm{KCl}, 1 \mathrm{mM}$ EDTA, $1 \mathrm{mM}$ dithiothreitol, $0.5 \mathrm{mM}$ phenylmethylsulfonyl fluoride, $10 \mu \mathrm{g} / \mathrm{mL}$ leupeptin, $40 \mu \mathrm{g} / \mathrm{mL}$ aprotinin, and $10 \mu \mathrm{g} / \mathrm{mL}$ pepstatin). Cell membranes were broken with a Dounce homogenizer using Pestle B. Nuclei were pelleted by centrifugation at $2000 \mathrm{~g}$ for 10 minutes at $4^{\circ} \mathrm{C}$ and washed with hypotonic buffer. Pellets were resuspended in high salt extraction buffer $(20 \mathrm{mM}$ Tris- $\mathrm{HCl}, \mathrm{pH} 7.5,420 \mathrm{mM} \mathrm{KCl}, 25 \%$ glycerol, $0.2 \mathrm{mM}$ EDTA, $0.5 \mathrm{mM}$ $\mathrm{MgCl}_{2}, 1 \mathrm{mM}$ dithiothreitol, $0.5 \mathrm{mM}$ phenylmethylsulfonyl fluoride, 10 $\mu \mathrm{g} / \mathrm{mL}$ leupeptin, $40 \mu \mathrm{g} / \mathrm{mL}$ aprotinin, and $10 \mu \mathrm{g} / \mathrm{mL}$ pepstatin), mixed vigorously by vortexing, and placed on ice for 15 minutes followed by centrifugation at $15000 \mathrm{~g}$ for 15 minutes at $4^{\circ} \mathrm{C}$. Supernatants (nuclear extracts) were stored in aliquots at $-80^{\circ} \mathrm{C}$. Samples were examined for degradation by SDS-polyacrylamide gel electrophoresis (PAGE). Protein concentration was determined by colorimetric assay (Bio-Rad Laboratories). Human adult normal liver nuclear protein and Swiss Webster mouse hepatocyte nuclear extract were also purchased from BioChain Institute (Hayward, CA). Nuclear extracts used to evaluate the effects of sex hormones on concentration and binding of nuclear proteins were derived from liver of C57B1/6 male and female mice, 2 weeks after castration or subcutaneous implantation of DHT pellets, respectively, by BioChain Institute.

\section{Gel mobility shift assay (GMSA)}

A series of dephosphorylated oligonucleotides encompassing the AAV-2 ITR sequences were used for GMSA and consisted of the following oligonucleotides: oligonucleotide 1a, 5'-TTGGCCACTC CCTCTCTGCG CGCTCGCTCG-3'; oligonucleotide 3a, 5'-CTCACTGAGG CCGGGCGACC AAAGGTCGCC-3'; oligonucleotide 5a, 5' -CGACGCCCGG GCTTTGCCCG GGCGGCCTCA-3'; oligonucleotide 7a, 5'-GTGAGCGAGC GAGCGCGCAG AGAGGGAGTG-3'; oligonucleotide 9a, 5'-GCCAACTCCA TCACTAGGGG TGTAGTTAAT-3'; oligonucleotide D-, 5'-AGGAACCCCTAGTGATGGAG-3'. The oligonucleotides were $5^{\prime}$ endlabeled using T4 polynucleotide kinase and $\left[\gamma_{-}{ }^{32} \mathrm{P}\right] \mathrm{ATP}$ (adenosine triphosphate; $\left.3000 \mathrm{Ci} / \mathrm{mmol}\left[111 \times 10^{12} \mathrm{~Bq} / \mathrm{mmol}\right]\right)$ with an average activity of $0.5 \times 10^{5} \mathrm{cpm} / \mathrm{ng}$. Gel mobility shift assays were performed by incubating $0.5 \mathrm{ng}$ labeled probe with nuclear extract $(10 \mu \mathrm{g})$ and competing oligonucleotides in binding buffer (10 mM Tris- $\mathrm{HCl}, \mathrm{pH} 7.5,50 \mathrm{mM} \mathrm{NaCl}, 1 \mathrm{mM}$ dithiothreitol, $1 \mathrm{mM}$ EDTA, $5 \%$ glycerol, and $1 \mu \mathrm{g} / \mu \mathrm{L}$ poly $(\mathrm{dI} \cdot \mathrm{dC})$ ) at room temperature for 30 minutes. Mixtures were size-fractionated on a nondenaturing $4 \%$ polyacrylamide gel followed by drying prior to autoradiography. The intensity of the complexes was determined using the STORM phosphorimager as in "Nucleic acid isolation and analysis."

\section{Results}

Sex difference in rAAV-mediated transgene expression after liver-targeted delivery of vector is independent of promoter and proviral cDNA

Hepatocyte transduction efficiencies were compared in cohorts of male and female B6.CB17-Prkdc-SzJ immunodeficient mice using an AAV-2-based vector (rAAV-2 CAGG-FIX; Figure 1A) encoding the human FIX cDNA under the control of the cytomegalovirus (CMV) enhancer, $\beta$-actin promoter. Equivalent numbers of vector genomes $\left(1 \times 10^{11} \mu \mathrm{g} /\right.$ mouse $)$, derived from the same batch of heparin affinity-purified vector stock, were administered into the portal vein of 7- to 10-week-old animals. As shown in Figure 1B, the kinetics of AAV transgene expression was similar in both male and female mice. However, plasma levels of hFIX were between 5and 13-fold higher in males compared with female mice with 

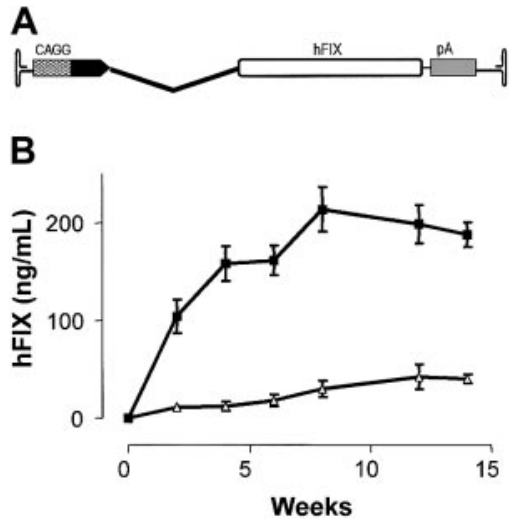

Figure 1. Influence of sex on rAAV-mediated hFIX expression. (A) Schematic of rAAV-2 CAGG-FIX consisting of CMV-IE enhancer, $\beta$-actin promoter, a chicken $\beta$-actin/rabbit $\beta$-globin composite intron (CAGG), 1.6-kb human FIX cDNA (hFIX), and a rabbit $\beta$-globin polyadenylation signal (hatched box) flanked by the AAV internal terminal repeats (ITR shown as hairpin loop). (B) Human FIX concentration (hFIX) in murine plasma after liver-directed administration of $1 \times 10^{11}$ rAAV CAGG-FIX in cohorts $(n=6)$ of male $(\square)$ and female $(\triangle)$ B6.CB17-Prkdc-SzJ mice. All transgene expression values are depicted as average together with the standard error of the mean.

steady-state levels of $213 \pm 23 \mathrm{ng} / \mathrm{mL}$ and $30 \pm 8 \mathrm{ng} / \mathrm{mL}$, respectively. The sex-specific difference in hFIX expression was highly significant at all time points $(P<.001$, one-way ANOVA) and reproducible with different stocks of vector in C.B-17 SCID and C57B1/6 immunocompetent strains of mice.

The minimal hFIX promoter and $5^{\prime}$ untranslated region $(-220$ to +43 ) contain androgen responsive elements, ${ }^{37,38}$ thus raising the possibility that enhanced hFIX expression in males was mediated by sequences retained in the hFIX cDNA within the rAAV-2 CAGG-FIX expression cassette ( +1 to $+43 \mathrm{bp})$. However, portal vein infusion of an rAAV vector (rAAV-2 CAGG-tsFlk, Figure 2A) in which the $h F I X$ gene was replaced with truncated soluble vascular endothelial growth factor receptor (tsFlk-1) cDNA also resulted in higher stable tsFlk-1 expression in male $(25 \pm 0.6$ $\mu \mathrm{g} / \mathrm{mL})$ compared with female $(5 \pm 0.1 \mu \mathrm{g} / \mathrm{mL})$ B6.CB17-PrkdcSzJ mice. The sex difference in tsFlk-1 expression was highly significant $(P<.001$, Student $t$ test $)$ and was observed in C.B-17 SCID and A/J immunocompetent mice. Replacement of the CAGG promoter with the $\alpha 1$-microglobulin/bikunin enhancer and thyroid hormone-binding globulin promoter (LSP) ${ }^{9}$ resulted in an almost 6-fold higher level of hFIX in male $(88 \pm 19 \mathrm{ng} / \mathrm{mL})$ compared with female $(15 \pm 5 \mathrm{ng} / \mathrm{mL}$ ) mice (Figure $2 \mathrm{~B}$ ), indicating that the mechanism that mediates higher rAAV transgene expression in male mice is not influenced by $\mathrm{rAAV}$ transgene promoter or cDNA.
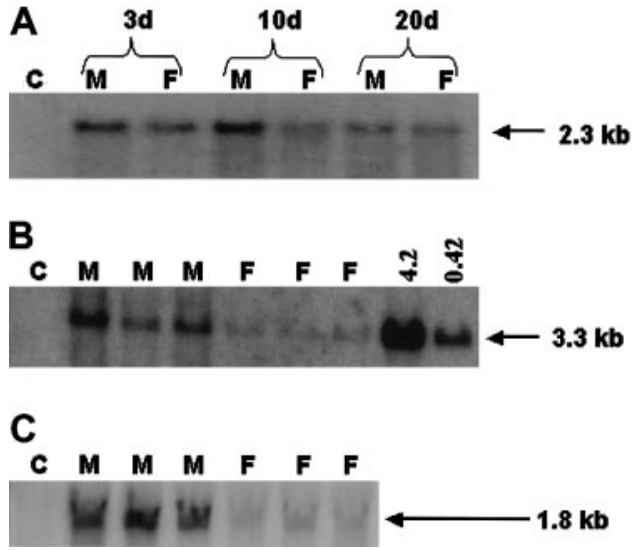

Figure 3. Molecular analysis of gene transfer efficiency in male and female mice. (A) Southern blot analysis of undigested low-molecular-weight Hirt DNA extracted from liver 3 days $(3 \mathrm{~d}), 10$ days $(10 \mathrm{~d})$, and 20 days $(20 \mathrm{~d})$ after portal vein infusion of vector particles. $C$ indicates DNA from naive control mouse; $M$ and $F$, DNA from rAAV CAGG-FIX transduced male and female mice, respectively. (B) Southern blot analysis of high-molecular-weight genomic DNA isolated from the liver 10 weeks after portal vein infusion of $1 \times 10^{11}$ rAAV-2 CAGG-FIX vector and digested with EcoRI and Hindll to release a 3.3-kb fragment from the expression cassette. $C$ indicates DNA from naive control mouse; $M$ and $F$, DNA from rAAV CAGG-FIX transduced male and female mice, respectively. The final 2 right-hand lanes consist of control mouse DNA spiked with 4.2 and 0.42 copies per diploid genome of plasmid pAV CAGG-FIX digested with EcoRI and HindIII. (C) Northern blot analysis of tota cellular RNA isolated from liver of male $(\mathrm{M})$ and female $(F)$ mice, 10 weeks following portal vein infusion of $1 \times 10^{11} \mathrm{rAAV}$ CAGG-FIX vector. $\mathrm{C}$ indicates RNA from naive control male mouse. For each of the above procedures, equivalent loading of nucleic acid on the respective gels was documented using Alphalmager software version 3.24 (Alpha Innotech).

\section{Molecular analysis of the AAV transgene in the liver}

To establish a mechanism for the sex difference in transgene expression, low-molecular-weight Hirt DNA was purified from the livers of male and female mice at different time points after liver-targeted delivery of $1 \times 10^{11}$ rAAV-2 CAGG-FIX vector particles. Southern blot analysis of undigested Hirt DNA revealed predominantly single-stranded vector genome at all the early time points assessed (days 3-20 after vector infusion) in samples derived from male and female liver. The relative level of single-stranded vector genome was significantly higher in male compared with female mice at 3, 10, and 20 days after portal vein infusion of vector particles (Figure 3A), consistent with sex-specific differences in attachment/endocytosis or trafficking of virions to the nucleus. However, as single-stranded genomes are unable to mediate expression, we next determined double-stranded rAAV copy number in murine livers, 10 weeks after portal vein infusion
A
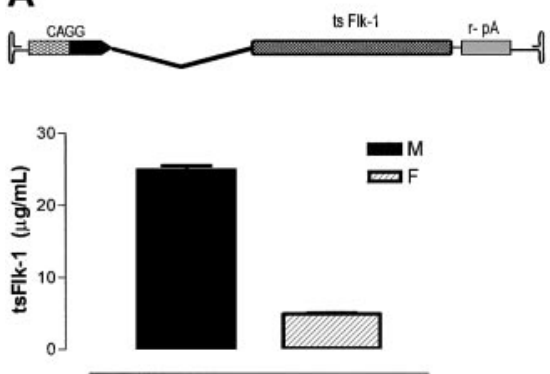

B
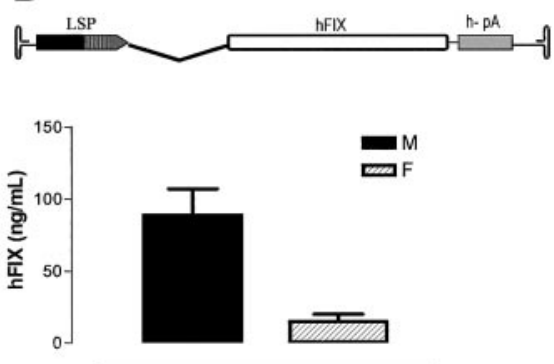

Figure 2. Sex difference in rAAV expression is independent of transgene cDNA and promoter. (A) Schematic of rAAV-2 CAGG tsFIk-1 in which the hFIX cDNA of rAAV-2 CAGG-FIX has been replaced with a 2.3-kb tsFlk-1 cDNA (top panel). Stable serum levels of tsFlk-1 assessed by ELISA, 12 weeks after portal vein infusion of $1 \times 10^{11}$ rAAV-2 CAGG tsFlk-1 vector particles in cohorts $(n=6)$ of male $(M)$ and female $(F)$ B6.CB17-Prkdc-SzJ mice (bottom panel). (B) Schematic of rAAV LSP-FIX in which the CAGG promoter has been replaced with an LSP (top panel). Stable plasma hFIX levels 12 weeks after portal vein infusion of $1 \times 10^{11} \mathrm{rAAV} L \mathrm{LP}$-FIX vector particles in cohorts $(\mathrm{n}=3$ ) of male $(\mathrm{M})$ and female $(\mathrm{F})$ B6.CB17-Prkdc-SzJ mice (bottom panel). All transgene expression values are depicted as an average together with the standard error of the mean. 
of $1 \times 10^{11} \mathrm{rAAV}-2 \mathrm{CAGG}-\mathrm{FIX}$. Southern blot analysis of equivalent amounts of high-molecular-weight genomic DNA digested with HindIII and SpeI to release an internal fragment of $3.3 \mathrm{~kb}$ containing the hFIX cDNA is shown in Figure 3B. Although we are unable to deduce absolute copy number for individual samples because of the lack of extensive standards, the relative difference in AAV transgene copy number between male and female mice was 7-fold and correlated closely with plasma hFIX expression in each cohort of mice. Northern blot analysis was performed to determine hFIX mRNA levels in murine liver 10 weeks after portal vein administration of $1 \times 10^{11}$ rAAV-2 CAGG-FIX vector particles, taking appropriate precaution to ensure equivalent amounts of intact total RNA was loaded for each sample. As demonstrated in Figure 3C, hFIX mRNA levels in murine liver were on average 6-fold higher in male compared with female mice, consistent with differential transduction at the genomic level.

\section{Influence of rAAV serotype and target tissue on differential rAAV expression based on sex}

AAV-2 vectors require $\mathrm{HPG}^{39}$ together with basic fibroblast growth factor receptor- 1 (FGFR-1 $)^{40}$ and $\alpha \mathrm{V} \beta 5$ integrin ${ }^{16}$ coreceptors for attachment and internalization of virions. Evaluation of FGFR-1 by Western blot analysis revealed similar levels of this coreceptor in murine liver irrespective of sex (data not shown). We next evaluated transduction efficiency with AAV-5-based vectors that rely on cellular 2,3-linked sialic acid for binding and internalization instead of HPG. As shown in Figure 4A, tail vein administration of $1 \times 10^{11}$ rAAV-5 CAGG-FIX vector particles that contained the CAGG-FIX expression cassette flanked by AAV-5 ITRs resulted in hFIX expression that was 6-fold higher in male $(102 \pm 27 \mathrm{ng} / \mathrm{mL})$ compared with female mice $(16 \pm 3 \mathrm{ng} / \mathrm{mL})$ at 6 weeks after gene transfer. The sex difference in expression observed with rAAV-5

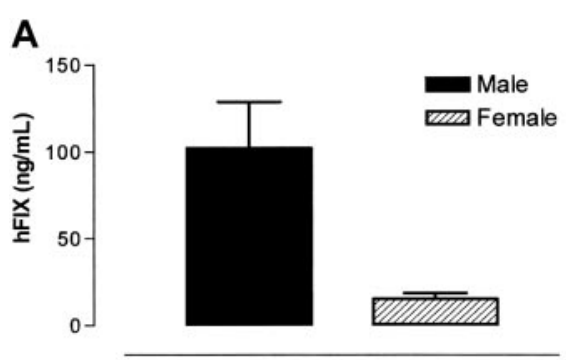

B

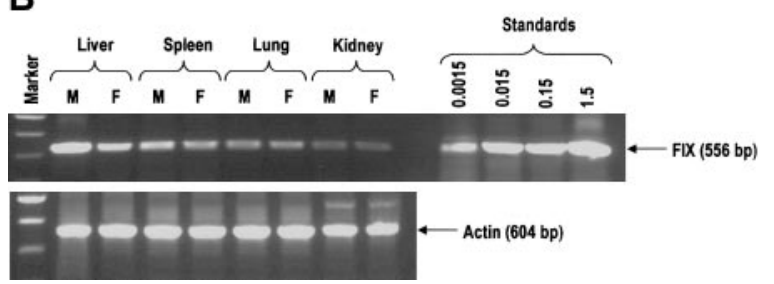

Figure 4. Influence of rAAV serotype and route of administration on sexmediated discordance in rAAV transgene expression. (A) Stable hFIX expression 6 weeks after tail vein infusion of $1 \times 10^{11}$ rAAV-5 CAGG-FIX, in a cohort $(n=3)$ of male and female B6.CB17-Prkdc-SzJ mice. Error bars indicate SEM. (B) Genomic DNA was extracted from liver, spleen, lungs, and kidney from male $(M)$ and female $(F)$ mice 6 weeks after tail vein administration of $1 \times 10^{11}$ rAAV-5 CAGG-FIX. Genomic DNA $(0.5 \mu \mathrm{g})$ was used for PCR amplification using transgene-specific primers designed to amplify a 521-bp product. Proviral copy number was deduced from standards, which consisted of serial dilutions of vector DNA $\left(1.5 \times 10^{-3}\right.$ to 1.5 copies) in $0.5 \mu \mathrm{g}$ control genomic DNA. Integrity of DNA was determined by amplifying a 604-bp region of murine $\beta$-actin gene and is shown at the bottom of the panel.
A

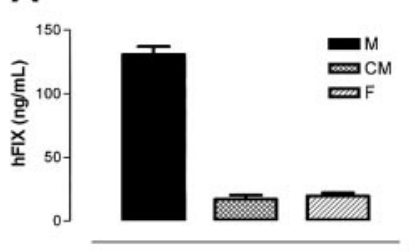

B
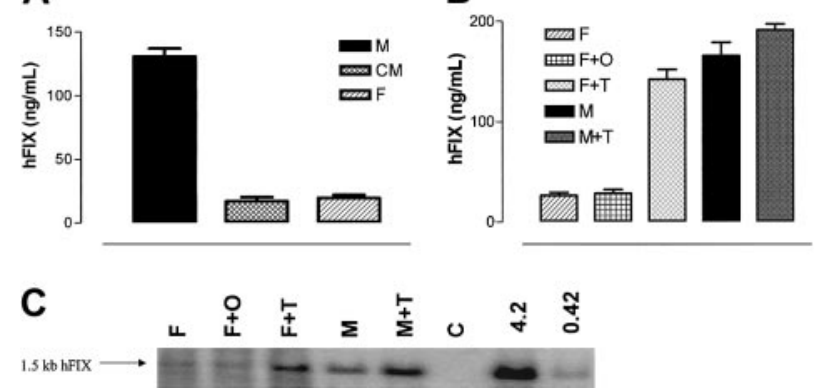

Figure 5. Effect of androgens on rAAV-mediated transgene expression. (A) Human FIX expression in C57BI/6 competent mice 6 weeks after tail vein administration of $1 \times 10^{11}$ rAAV-2 CAGG-FIX vector particles. $M$ indicates naive males $(n=3)$; CM, castrated animals $(n=5) ; F$, naive females $(n=3)$. Transgene expression values represent means together with the standard error for each cohort. (B) Stable hFIX levels were determined 10 weeks after tail vein administration of $1 \times 10^{11}$ rAAV-2 CAGG-FIX vector particles in unmanipulated female mice $(F)$ female mice that had been oophorectomized 2 weeks prior to vector administration $(\mathrm{F}+\mathrm{O})$, oophorectomized female mice treated with $\mathrm{DHT}$ prior to vector administration $(F+T)$, naive male mice $(M)$, and male mice primed with DHT prior to vector administration $(\mathrm{M}+\mathrm{T})$. Human FIX expression values are depicted as average together with the standard error of the mean of each cohort containing between 3 to 7 mice. (C) Southern blot analysis of DNA isolated from murine liver 10 weeks after tail vein administration $1 \times 10^{11} \mathrm{rAAV}$ CAGG-FIX vector particles. DNA samples were digested with $N$ hel to release an internal fragment of $1.5 \mathrm{~kb}$. Sample order from right to left is as follows: lane 1, unmanipulated female mouse $(F)$; lane 2, oophorectomized mouse $(F+O)$; lane 3, oophorectomized mouse treated with DHT $(F+T)$ lane 4, naive male mouse (M); lane 5, male mouse primed with $\mathrm{DHT}(\mathrm{M}+\mathrm{T})$; lane 6 , DNA from a mock-transduced male mouse. Lanes 7 and 8 are control mouse DNA spiked with 4.2 and 0.42 copies per diploid genome of plasmid pAV CAGG-FIX digested with Nhel.

CAGG-FIX is similar to that seen after liver-targeted delivery of AAV-2-based vectors. The biodistribution of the AAV 5 genome after tail vein administration of vector particles was next determined by a semiquantitative PCR assay capable of detecting $1.5 \times 10^{-3}$ copies per diploid genome (CPDG). The rAAV-5 CAGG-FIX genome was detectable in all tissue samples examined with disproportionately higher levels in the liver (Figure 4B), as previously described with AAV-2-based vectors. ${ }^{4}$ The proviral copy number was, however, 3-fold higher in male (0.57 CPDG) compared with female liver (0.18 CPDG). In contrast, comparable gene transfer efficiencies were observed in spleen (0.01 CPDG in males and females), lungs (0.09 and 0.07 CPDG in males and females, respectively), and kidneys (0.03 and 0.04 CPDG in males and females, respectively) derived from individual male and female mice. Furthermore, injection of $1 \times 10^{11} \mathrm{rAAV}-2$ CAGG-FIX vector particles directly into the hind limb skeletal muscle of B6.CB17Prkdc-SzJ mice resulted in equivalent levels of transgene expression in male $(63 \pm 6 \mathrm{ng} / \mathrm{mL})$ and female $(60 \pm 5 \mathrm{ng} / \mathrm{mL})$ mice.

Thus, the sex-specific difference in transgene expression is observed with AAV serotypes that use different receptors for attachment and endocytosis. Additionally, there is a direct correlation between differential transgene expression and in vivo transduction of hepatocytes. Intriguingly, AAV-mediated gene transfer into nonhepatic organs is not significantly influenced by sex, indicating that the biologic processing of AAV in the liver involves mechanisms that are distinct from those in nonhepatic organs.

\section{Androgens enhance rAAV transduction efficiency after liver-targeted delivery of vector particles}

Given the significantly higher transgene expression in male mice following liver-targeted delivery of rAAV vector, we sought to investigate the effect of castration on AAV transduction efficiency. 
As shown in Figure 5A, tail vein administration of $1 \times 10^{11}$ rAAV-2 CAGG-FIX vector into unmanipulated C57B1/6 immunocompetent mice resulted in hFIX expression at $131 \pm 6 \mathrm{ng} / \mathrm{mL}$ in a cohort of males compared with $20 \pm 2 \mathrm{ng} / \mathrm{mL}$ in female mice at 6 weeks, consistent with the sex-specific difference in transgene expression after portal vein administration of AAV-2-based vector. Castration 2 weeks prior to vector tail vein administration of an equivalent dose of vector derived from the same stock of rAAV-2 CAGG-FIX substantially decreased hFIX expression $(17 \pm 3 \mathrm{ng} /$ $\mathrm{mL}$ ) to levels observed in female mice. These results strongly indicate that androgens are responsible for mediating efficient transduction in males.

As with $\mathrm{C} 57 \mathrm{~B} 1 / 6$ immunocompetent mice, administration of $1 \times 10^{11}$ rAAV-2 CAGG-FIX vector particles into the tail vein of B6.CB17-Prkdc-SzJ mice (Figure 5B) resulted in 6-fold higher levels of hFIX in males (166 $\pm 3 \mathrm{ng} / \mathrm{mL})$ compared with female mice $(26 \pm 3 \mathrm{ng} / \mathrm{mL})$. Oophorectomy performed either before or after $\mathrm{AAAV}$ vector infusion did not result in a significant change in rAAV-mediated transgene expression, implying that estrogens did not influence AAV transduction. In contrast, subcutaneous administration of androgens in the form of slow-release DHT pellets, 2 weeks prior to gene transfer, increased hFIX expression in oophorectomized female mice by 5 -fold $(142 \pm 10 \mathrm{ng} / \mathrm{mL})$ to levels observed in males (Figure 5B). The timing of androgen administration was critical, as subcutaneous implantation of DHT at the time of or after rAAV delivery did not augment transgene expression in females (data not shown). Interestingly, administration of DHT in male mice 2 weeks prior to tail vein injection of rAAV-2 CAGG-FIX enhanced hFIX expression mice by approximately $15 \%$ (Figure 5B), indicating that rAAV expression could be augmented in males with supplemental androgens. Importantly, the enhanced expression observed in DHT-treated animals was persistent for the duration of the study ( $>6$ months) in the absence of further supplementation with androgens in mice of both sexes.

At a molecular level, rAAV transgene copy number in female mice increased from a baseline of 0.2 copies per diploid genome in naive animals to 0.8 copies per diploid genome in mice treated with DHT prior to vector administration (Figure 5C). Oophorectomy did not result in a significant change in gene transfer. However, a 3-fold increase in transduction efficiency was observed in male mice (1.5 copies per diploid genome) that received DHT prior to gene transfer when compared with controls ( 0.5 copies per diploid genome).

\section{AAV transgene expression by human cells is enhanced by androgens}

Previous reports indicated that HPG and FGFR-1, which serve as receptors for AAV-2, could be up-regulated by androgens. ${ }^{41}$ To establish if androgens enhanced gene transfer into hepatocyte by increasing binding and uptake of $\mathrm{AAV}$, we turned to a human hepatocellular carcinoma cell line ( $\mathrm{HuH} 7),{ }^{35}$ derived from a male patient. Transduction of $\mathrm{HuH} 7$ cells with rAAV-2 CAGG-FIX at an MOI of $10^{3}$ resulted in hFIX expression at a level of $218 \pm 4$ $\mathrm{ng} / \mathrm{mL}(\mathrm{n}=5)$ in conditioned media over a 24-hour period. Addition of DHT to cell culture media at a concentration of $300 \mathrm{nM}$ 5 days prior to transduction with $\mathrm{rAAV}-2 \mathrm{CAGG}-\mathrm{FIX}$ resulted in an increase $(25 \%)$ in hFIX expression to $271 \pm 12 \mathrm{ng} / \mathrm{mL}$, comparable to the increase in transgene expression observed in male mice exposed to supplemental androgens (Figure 6A). Binding of AAV-2 particles labeled with $\mathrm{Cy} 3$ fluorescent dye to unmanipulated and DHT-treated HuH7 cells at an MOI of $10^{3}$ was broadly similar as assessed by the mean fluorescent intensity over several fields by confocal microscopy (Figure 6B). Specificity of binding was

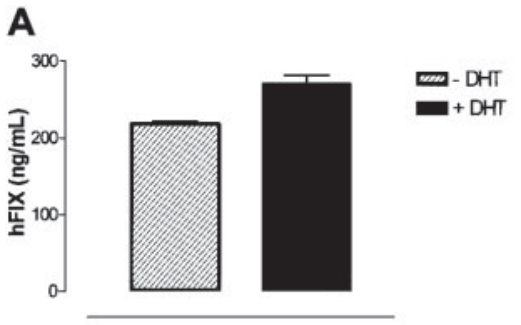

B
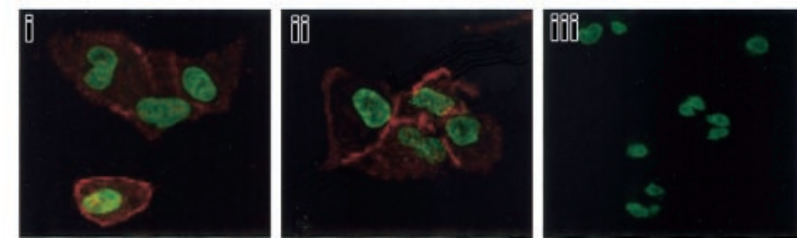

Figure 6. Effects of androgens on rAAV transduction and attachment to human HuH7 cell line. (A) Human $\mathrm{HuH} 7$ hepatocarcinoma cells were cultured in serum-free hepatocyte culture media with (+DHT) or without ( $-\mathrm{DHT}) 300 \mathrm{nM}$ of DHT for 5 days prior to gene transfer. Cells from each treatment group $(n=5)$ were plated out at a density of $1 \times 10^{5}$ cells per well of 6 -well plates and transduced at an $\mathrm{MOI}$ of $10^{3}$ with rAAV-2 CAGG-FIX. Medium was conditioned for 24 hours and collected on day 4 after transduction and assayed for hFIX by ELISA. Error bars indicate SEM. (B) Fluorescence confocal micrographs of optical sections demonstrating attachment of Cy-3 (red fluorescence) labeled rAAV-2 CAGG-FIX to (i) untreated $\mathrm{HuH} 7$ cells, (ii) $\mathrm{HuH} 7$ cells treated with $300 \mathrm{nM} / \mathrm{L}$ DHT for 5 days, or (iii) CHO-K1 cells that lack HPG. The nuclei show a low level of green autofluorescence that was enhanced in these images simply to identify the whole cell. Original magnification, $\times 335$ (i-ii) and $\times 178$ (iii)

demonstrated by the absence of attachment of labeled virions to CHO-K1 cells that are deficient in HPG.

\section{Interaction of host nuclear proteins with the AAV ITR may be responsible for the higher rAAV gene transfer efficiency in males}

We hypothesized that the observed sex difference in transduction efficiency was mediated by differential binding of testosteronedependent host nuclear regulatory protein(s) to specific sequences within the AAV ITR. To test this hypothesis, purified nuclear extracts from male and female murine livers were used in a competitive gel mobility shift assay with a series of overlapping radiolabeled single-stranded oligonucleotides spanning the AAV-2 ITR (Figure 7A). Oligonucleotide 7 (Oligo 7) that encompassed the RBS formed strong specific complexes with both male and female nuclear extracts that could be abolished with 50-fold molar excess of unlabeled cognate (Figure 7B). The intensity of the complex formed with male hepatocyte nuclear extracts was 4-fold higher than that observed with an equivalent amount of female hepatocyte nuclear proteins, consistent with the sex-specific difference in transduction efficiency. Differential binding was also observed with oligonucleotides 1 and 3 , which overlap oligonucleotide 7 , although the intensity of the complexes formed with these oligonucleotides was significantly lower $(7>1>3$, data not shown). Relatively weaker complexes of roughly equivalent intensity were observed with an oligonucleotide (oligo D-), containing the minus strand D AAV ITR sequences that have previously been shown to bind a host protein tyrosine kinase in a mechanism that regulates AAV second strand synthesis. ${ }^{25}$ DNA-nuclear protein interactions were not observed with other AAV ITR sequences such as oligonucleotide 5 (oligo 5), and in addition the observed pattern of DNA-protein binding was reproducible with different stocks of mouse liver nuclear extracts.

To determine whether androgens could influence the concentration or binding affinity of the host RBS binding protein, nuclear 
A

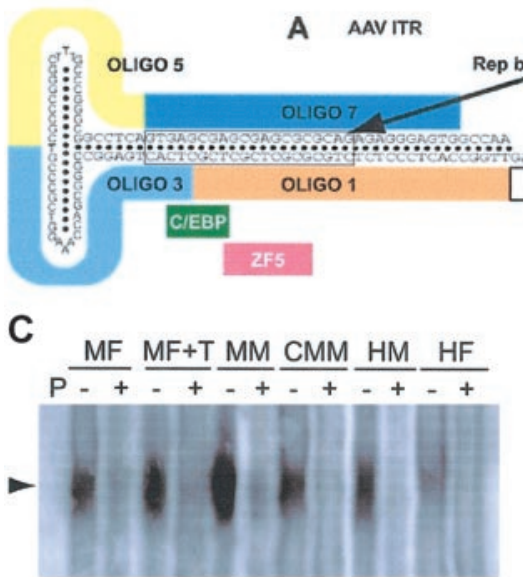

B

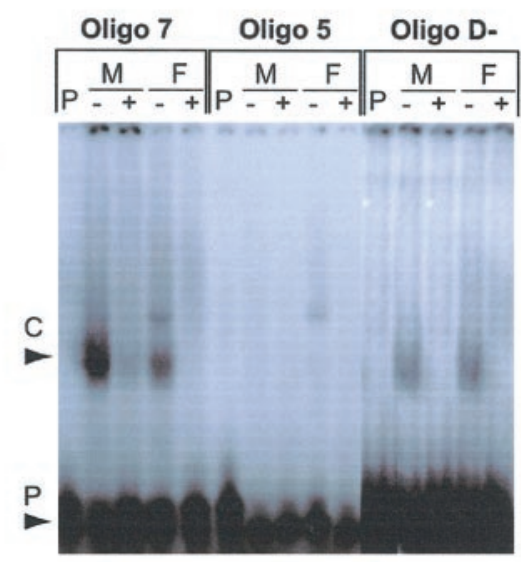

Figure 7. Competitive gel mobility shift assay. (A) Schematic of the AAV-2 ITR demonstrating the positions of the Rep-binding site as well as the pertinent 30 meric oligonucleotides. The location of the consensus sequence for CCAAT/enhancer binding protein alpha (C/EBP) and human zinc finger 5 protein (ZF5) is also shown. (B) Gel mobility shift assay performed with equivalent amounts of nuclear protein derived from the liver of male and female mice and radiolabeled 30 meric single-stranded oligonucleotides encompassing the A region (oligo 7) include the rep binding site, $\mathrm{C}$ region (oligo 5), and the D- region (oligo D-) in the presence (+) or absence (-) of a 50-M excess of unlabeled cognate. Retarded nuclear DNA complex (C) and free radiolabeled probe (P) are indicated by arrows. (C) Gel mobility shift assay demonstrating the intensity of binding to radiolabeled oligonucleotide 7 with equivalent amounts of hepatocyte nuclear extracts derived from murine (MF indicates naive female; MF + DHT, female pretreated with $\mathrm{DHT}$; MM, unmanipulated male; CMM, castrated male mouse) and human donors (HM indicates male; HF, female) in the presence (+) or absence (-) of a 50-M excess of unlabeled cognate. Retarded nuclear DNA complex (C) is indicated by the arrow.

extracts were prepared from the liver of female and male mice 2 weeks after pretreatment with slow release DHT or castration, respectively, and used in a gel mobility shift assay with oligo 7. As before, the intensity of the DNA-nuclear protein complexes formed with nuclear extract from naive male liver was substantially higher than with female cognate (Figure 7C). Binding of nuclear proteins derived from the hepatocytes of females treated with supplemental testosterone was significantly greater (1.5-fold) than that observed with an equivalent amount of extract from naive female mice. Castration reduced the intensity of the DNA-nuclear protein complex by 5 -fold when compared with the complexes formed with nuclear extracts derived from the liver of unmanipulated male mice. These results indicate that androgens have a direct effect on the concentration and/or binding affinity of the host RBS protein in concordance with the sex differences in AAV expression.

Gel shift analysis with normal human adult liver nuclear extracts and radiolabeled oligonucleotide 7 revealed complexes that had the same migration pattern as that observed with murine hepatocyte extracts (Figure 7C). In concurrently run samples, the intensity of these complexes was significantly lower than that observed with an equivalent amount of murine cognate irrespective of sex. This finding suggests that the concentration or binding affinity of RBS binding host protein in human liver is lower than that in mice. Importantly, the intensity of the DNA-nuclear protein complex formed with human hepatocytes showed a significant sex bias with significantly higher binding (1.8-fold) with male extracts when compared with female cognate, consistent with the difference observed in mice.

\section{Discussion}

This study represents the first systematic evaluation of the influence of sex on rAAV-mediated gene transfer. Our results indicate that rAAV gene transfer to hepatocytes is strongly influenced by sex. Liver-targeted delivery of AAV vector particles resulted in significantly higher transgene expression in male mice compared with female mice irrespective of the proviral promoter or cDNA. Castration reduced AAV transgene expression in males to levels observed in female mice, whereas transient exposure of female mice to DHT prior to administration of rAAV vector improved stable hepatocyte gene transfer levels observed in males, suggesting that androgens are responsible for the enhanced gene transfer. Oophorectomy had no effect on rAAV gene transfer. The increase in rAAV transduction of the liver was observed only when DHT was administered a few days prior to vector infusion, indicating that the cellular machinery had to be primed for optimal gene transfer with rAAV vectors. The sex difference in transduction was observed with AAV-2- and AAV-5-based vectors, which use distinct receptor complexes for infection and could not be attributed to differential attachment of virion to testosterone-treated hepatocytes. Specific and disparate binding of the AAV-2 ITR sequences encompassing the RBS was observed with host nuclear proteins derived from male and female liver in both mice and humans, which correlated strongly with the sex difference in AAV-mediated transduction. The specific binding of the host nuclear RBS protein was moderated by androgens, indicating that it was integrally involved in the androgen-dependent mechanism responsible for differential transduction of the liver.

Sex-specific differences in susceptibility to infection by wildtype AAV of the respiratory system have not been reported. ${ }^{42}$ Indeed, our study demonstrates that gene transfer into nonhepatic organs, including the lung, is not influenced by sex. This finding suggests that the mechanisms responsible for efficient transduction of the liver, an organ that is not naturally infected by AAV, are distinct from those in nonhepatic tissues. Others whose results have been reported in abstract form have also found sex differences in rAAV transduction, ${ }^{43,44}$ although ours is the first study to critically evaluate the mechanisms responsible for the sex-specific differences in AAV transgene expression.

Androgens up-regulate the AAV-2 receptors, HPG, and FGFR-1 in some tissues, ${ }^{41}$ raising this as a possible mechanism for sex-specific differences in transduction of the liver. However, FGFR-1 levels in liver tissue derived from male and female mice 
were comparable. In addition, differential attachment by $\mathrm{Cy} 3$ labeled rAAV-2 CAGG-FIX vector particles to testosterone-treated $\mathrm{HuH} 7$ cells was not observed, whereas transgene expression in these cells was clearly augmented by androgens, although we accept that minor differences in attachment of the fluorescentlabeled vector may not be apparent. Furthermore, the sex difference in transduction was observed with AAV-5 vectors, which use cell surface 2,3-linked sialic acid for infection. ${ }^{45}$ Up-regulation of cellular 2,3-linked sialic acid by androgens has not been reported. Finally, augmentation of AAV trafficking by androgens is unlikely to be the mechanism that mediates higher transduction of the liver in males, as this sex hormone enhances endosomal degradation of proteins. ${ }^{46-48}$ Thus, our combined data suggest alternative mechanisms are responsible for the sex difference in transduction of liver.

It is widely acknowledged that host proteins are required for stable rAAV transduction. Indeed, interaction of a host nuclear protein, ssD-BP, with the $\mathrm{D}-$ region of the AAV ITR has been shown to influence transduction. ${ }^{25}$ We, however, did not observe any difference in the binding of the AAV-2 D- region with hepatocyte nuclear extracts derived from male and female liver. In contrast, binding of the hepatocyte nuclear proteins to AAV-2 ITR sequences comprising the RBS was significantly higher in males compared with females in both mice and humans. Additionally, the concentration and or the binding affinity of this host RBS nuclear protein in murine livers was reduced by castration but enhanced, in females, by supplemental androgens. The differential binding pattern observed with RBS correlates closely with AAV-mediated gene transfer in the liver, strongly suggesting that these 2 observations are causally linked. The Rep proteins through their interaction with the RBS present on the AAV ITR, p5 promoter, and the AAVS1 integration locus are responsible for regulating replication of the wild-type AAV genome ${ }^{49}$ and mediate site-specific integration into chromosome $19 .{ }^{50}$ Our data suggest a novel role for the AAV RBS in that its interaction with a distinct hepatocellular nuclear protein, whose expression or binding affinity is regulated by androgens, is critical for stable transduction of the liver, possibly by a mechanism that enhances AAV second strand synthesis or facilitates intermolecular recombination of the complementary AAV genomes.

The identity of the hepatocyte nuclear protein that interacts with the AAV ITR RBS remains obscure. ZF5 and C/EBP- $\alpha$ are potential candidates on the basis of the presence of highly conserved consensus binding sequences in RBS of both AAV-2 and AAV-5 (Figure 7). As ZF5 impairs replication of wild-type AAV and production of recombinant vectors, ${ }^{51}$ it is highly improbable that it is involved in the mechanisms that lead to sex-specific differences in AAV transduction of the liver. $\mathrm{C} / \mathrm{EBP}-\alpha$ is involved in the regulation of liver-specific genes, hepatitis $\mathrm{B}$ virus proliferation, and adipogenesis through an androgen-dependent pathway. ${ }^{52-55}$ However, the absence of specific complexes between the RBS and purified C/EBP protein and the lack of a supershift of the complex formed with murine hepatocyte nuclear extract by an anti-C/EBP polyclonal antibody (A.M.D. and A.C.N., unpublished observations, November 15, 2002) suggest that alternative host nuclear proteins are responsible for the androgen-specific binding pattern observed with RBS.

Further studies are required to unequivocally determine if the androgen-specific mechanism that leads to efficient in vivo transduction of the murine is active in other species, including humans. A recent report by Mount et $\mathrm{al}^{12}$ demonstrated similar levels of canine FIX in male and female dogs with hemophilia B after liver-targeted delivery of an equivalent number of rAAV particles encoding the canine $F I X$ gene. But firm conclusions cannot be drawn from this study, as apart from the small number of animals, the male and female dogs were of different strains, and AAV preparations injected into these animals were derived from different stocks with potentially different biologic activity. Our data with $\mathrm{HuH} 7$ cells and primary human hepatocytes strongly suggest that transduction of the human liver by AAV vectors will be moderated by an androgen-dependent pathway. This will undoubtedly have a significant effect on gene therapy strategies with AAV vectors that target the liver as females and juvenile male patients are likely to have a substantially lower transduction efficiency than males. However, on the basis of our data, it is likely that the barriers to efficient AAV-mediated transduction in these individuals could be overcome by a short course of androgens.

The mechanisms for efficient transduction with the rAAV system remain largely undefined despite the enormous interest in this vector system. We have unveiled a novel androgen-dependent pathway for augmenting hepatocyte gene transfer that may have significant implications for the translation of gene therapy to the clinic and merits further investigation.

\section{Acknowledgments}

We thank Jean Johnson for her outstanding assistance in preparation of the manuscript. The technical assistance of Dr K. G. Murti with the Cy-3-labeled AAV binding studies was invaluable. In addition, support from Dr Perdeep Mehta in the Hartwell Center for Bioinformatics and Biotechnology, St. Jude Children's Research Hospital, Memphis, TN, is greatly appreciated.

\section{References}

1. Nathwani AC, Davidoff AM, Hanawa H, et al. Sus tained high-level expression of human factor IX ( $\mathrm{hFIX)} \mathrm{after} \mathrm{liver-targeted} \mathrm{delivery} \mathrm{of} \mathrm{recombinan}$ adeno-associated virus encoding the hFIX gene in rhesus macaques. Blood. 2002;100:1662 1669.

2. Xiao W, Berta SC, Lu MM, Moscioni AD, Tazelaar $\mathrm{J}$, Wilson JM. Adeno-associated virus as a vecto for liver-directed gene therapy. J Virol. 1998;72: 10222-10226.

3. Daly TM, Okuyama T, Vogler C, Haskins ME, Muzyczka N, Sands MS. Neonatal intramuscular injection with recombinant adeno-associated virus results in prolonged beta-glucuronidase expression in situ and correction of liver pathology in mucopolysaccharidosis type VII mice. Hum Gene Ther. 1999;10:85-94.

4. Nathwani AC, Davidoff A, Hanawa H, Zhuo F, Va- nin EF, Nienhuis AW. Factors influencing in vivo transduction by recombinant adeno-associated viral vectors expressing the human factor IX cDNA. Blood. 2001;97:1258-1265.

5. Herzog RW, Hagstrom JN, Kung SH, et al. Stable gene transfer and expression of human blood coagulation factor IX after intramuscular injection of recombinant adeno-associated virus. Proc Natl Acad Sci U S A. 1997; 94:5804-5809.

6. Herzog RW, Yang EY, Couto LB, et al. Long-term correction of canine hemophilia B by gene transfer of blood coagulation factor IX mediated by adeno-associated viral vector. Nat Med. 1999;5:5663.

7. Snyder RO, Miao $\mathrm{CH}$, Patijn GA, et al. Persistent and therapeutic concentrations of human factor IX in mice after hepatic gene transfer of recombinant AAV vectors. Nat Genet. 1997;16:270-276.
8. Snyder RO, Miao C, Meuse L, et al. Correction of hemophilia $B$ in canine and murine models using recombinant adeno-associated viral vectors. Nat Med. 1999;5:64-70.

9. Wang L, Takabe K, Bidlingmaier SM, III CR, Verma IM. Sustained correction of bleeding disorder in hemophilia B mice by gene therapy. Proc Natl Acad Sci U S A. 1999;96:3906-3910.

10. Wang L, Nichols TC, Read MS, Bellinger DA, Verma IM. Sustained expression of therapeutic level of factor IX in hemophilia B dogs by AAVmediated gene therapy in liver. Mol Ther. 2000;1: 154-158.

11. Chao H, Samulski R, Bellinger D, Monahan $P$, Nichols T, Walsh C. Persistent expression of canine factor IX in hemophilia B canines. Gene Ther. 1999;6:1695-1704. 
12. Mount JD, Herzog RW, Tillson DM, et al. Sustained phenotypic correction of hemophilia B dogs with a factor IX null mutation by liverdirected gene therapy. Blood. 2002;99:26702676.

13. Chirmule N, Propert K, Magosin S, Qian Y, Qian $\mathrm{R}$, Wilson J. Immune responses to adenovirus and adeno-associated virus in humans. Gene Ther. 1999;6:1574-1583

14. Kay MA, Manno CS, Ragni MV, et al. Evidence for gene transfer and expression of factor IX in haemophilia B patients treated with an AAV vector. Nat Genet. 2000;24:257-261.

15. Kay MA, High K, Glader B, et al. A phase I/II clinical trial for liver directed AAV-mediated gene transfer for severe hemophilia B [abstract]. Blood. 2002;100:115a.

16. Summerford C, Bartlett JS, Samulski RJ. AlphaVbeta5 integrin: a co-receptor for adeno-associated virus type 2 infection. Nat Med. 1999;5:7882.

17. Zolotukhin S, Byrne BJ, Mason E, et al. Recombinant adeno-associated virus purification using novel methods improves infectious titer and yield. Gene Ther. 1999;6:973-985.

18. Nathwani AC, Hanawa H, Vandergriff J, Kelly P, Vanin EF, Nienhuis AW. Efficient gene transfer into human cord blood $\mathrm{CD} 34^{+}$cells and the CD $34^{+}$CD $38^{-}$subset using highly purified recombinant adeno-associated viral vector preparations that are free of helper virus and wild-type AAV. Gene Ther. 2000;7:183-195.

19. Duan D, Yue Y, Yan Z, Yang J, Engelhardt JF. Endosomal processing limits gene transfer to polarized airway epithelia by adeno-associated virus. J Clin Invest. 2000;105:1573-1587.

20. Douar AM, Poulard K, Stockholm D, Danos O. Intracellular trafficking of adeno-associated virus vectors: routing to the late endosomal compartment and proteasome degradation. J Virol. 2001 75:1824-1833.

21. Hansen J, Qing K, Kwon HJ, Mah C, Srivastava A. Impaired intracellular trafficking of adenoassociated virus type 2 vectors limits efficient transduction of murine fibroblasts. J Virol. 2000; 74:992-996.

22. Fisher KJ, Gao GP, Weitzman MD, DeMatteo R, Burda JF, Wilson JM. Transduction with recombinant adeno-associated virus for gene therapy is limited by leading-strand synthesis. J Virol. 1996; 70:520-532.

23. Ferrari FK, Samulski T, Shenk T, Samulski RJ. Second-strand synthesis is a rate-limiting step for efficient transduction by recombinant adenoassociated virus vectors. J Virol. 1996;70:32273234.

24. Hargrove PW, Vanin EF, Kurtzman GJ, Nienhuis AW. High-level globin gene expression mediated by a recombinant adeno-associated virus genome that contains the $3^{\prime}$ gamma globin gene regulatory element and integrates as tandem copies in erythroid cells. Blood. 1997;89:2167 2175 .

25. Mah C, Qing K, Khuntirat B, et al. Adeno-associ- ated virus type 2-mediated gene transfer: role of epidermal growth factor receptor protein tyrosine kinase in transgene expression. J Virol. 1998;72: 9835-9843.

26. Miao $\mathrm{CH}$, Nakai $\mathrm{H}$, Thompson $\mathrm{AR}$, et al. Nonrandom transduction of recombinant adeno-associated virus vectors in mouse hepatocytes in vivo: cell cycling does not influence hepatocyte transduction. J Virol. 2000;74:3793-3803.

27. Davidson BL, Stein CS, Heth JA, et al. Recombinant adeno-associated virus type 2, 4, and 5 vectors: transduction of variant cell types and regions in the mammalian central nervous system. Proc Natl Acad Sci U S A. 2000;97:3428-3432.

28. Ali RR, Reichel MB, Thrasher AJ, et al. Gene transfer into the mouse retina mediated by an adeno-associated viral vector. Hum Mol Genet. 1996;5:591-594.

29. Fisher KJ, Jooss K, Alston J, et al. Recombinant adeno-associated virus for muscle directed gene therapy. Nat Med. 1997;3:306-312.

30. Nakai H, Yant SR, Storm TA, Fuess S, Meuse L, Kay MA. Extrachromosomal recombinant adenoassociated virus vector genomes are primarily responsible for stable liver transduction in vivo. J Virol. 2001;75:6969-6976.

31. Davidoff AM, Nathwani AC, Spurbeck WW, Ng CY, Zhou J, Vanin EF. rAAV-mediated long-term liver-generated expression of an angiogenesis inhibitor can restrict renal tumor growth in mice. Cancer Res. 2002;62:3077-3083.

32. Chiorini JA, Kim F, Yang L, Kotin RM. Cloning and characterization of adeno-associated virus type 5. J Virol. 1999;73:1309-1319.

33. Auricchio A, O'Connor E, Hildinger M, Wilson JM. A single-step affinity column for purification of serotype- 5 based adeno-associated viral vectors. Mol Ther. 2001;4:372-374

34. Hirt B. Selective extraction of polyoma DNA from infected mouse cell cultures. J Mol Biol. 1967;26: 365-369.

35. Cichon G, Boeckh-Herwig S, Elezkurtaj S, Schmidt HH, Hofmann C, Arnold W. Hepatocarcinoma cells constitutively expressing adenovira E1-genes provide a tumor model for intratumora replication of E1-deficient adenoviruses. Anticancer Res. 2002;22:197-201.

36. Dignam JD, Lebovitz RM, Roeder RG. Accurate transcription initiation by RNA polymerase II in a soluble extract from isolated mammalian nuclei. Nucleic Acids Res. 1983;11:1475-1489.

37. Briet E, Wijnands MC, Veltkamp JJ. The prophylactic treatment of hemophilia B Leyden with anabolic steroids. Ann Intern Med. 1985;103:225 226.

38. Morgan GE, Rowley G, Green PM, Chisholm M, Giannelli F, Brownlee GG. Further evidence for the importance of an androgen response element in the factor IX promoter. Br J Haematol. 1997;98: 79-85.

39. Summerford C, Samulski RJ. Membrane-associated heparan sulfate proteoglycan is a receptor for adeno-associated virus type 2 virions. J Virol. 1998;72:1438-1445.
40. Qing K, Mah C, Hansen J, Zhou S, Dwarki V, Srivastava A. Human fibroblast growth factor receptor 1 is a co-receptor for infection by adenoassociated virus 2. Nat Med. 1999;5:71-77.

41. Ruohola JK, Valve EM, Vainikka S, Alitalo K Harkonen PL. Androgen and fibroblast growth factor (FGF) regulation of FGF receptors in S115 mouse mammary tumor cells. Endocrinology. 1995;136:2179-2188.

42. Monto AS. Epidemiology of viral respiratory infections. Am J Med. 2002;112(suppl 6A):4S-12S.

43. Reyes L, Alexander JJ, Song S, et al. The effect of sex hormones on rAAV-mediated gene therapy in female $\mathrm{Pah}{ }^{\text {enu2 }}$ mice, an animal model for PKU [abstract]. Mol Ther. 2001;3:S232-S233.

44. Lonning SM, Ford CA, Taylor KM, et al. Influence of gender-specific hormones on adeno-associated virus vector gene transfer and expression [abstract]. Mol Ther. 2002;5:S2.

45. Walters RW, Yi SM, Keshavjee S, et al. Binding of adeno-associated virus type 5 to 2,3-linked sialic acid is required for gene transfer. J Biol Chem. 2001;276:20610-20616.

46. Mezey E, Potter JJ. Effect of dihydrotestosterone on rat liver alcohol dehydrogenase activity. Hepatology. 1982;2:359-365.

47. Waltregny D, Leav I, Signoretti S, et al. Androgendriven prostate epithelial cell proliferation and differentiation in vivo involve the regulation of p27. Mol Endocrinol. 2001:15:765-782.

48. Taneja SS, Ha S, Garabedian MJ. Androgen stimulated cellular proliferation in the human prostate cancer cell line LNCaP is associated with reduced retinoblastoma protein expression. J Cell Biochem. 2001;84:188-199.

49. Labow MA, Hermonat PL, Berns KI. Positive and negative autoregulation of the adeno-associated virus type 2 genome. J Virol. 1986;60:251-258.

50. Kotin RM, Menninger JC, Ward DC, Berns KI Mapping and direct visualization of a regionspecific viral DNA integration site on chromosome 19q13-qter. Genomics. 1991;10:831-834.

51. Cathomen T, Stracker TH, Gilbert LB, Weitzman MD. A genetic screen identifies a cellular regulator of adeno-associated virus. Proc Natl Acad Sci U S A. 2001;98:14991-14996.

52. Davies N, Austen DE, Wilde MD, Darlington GJ, Brownlee GG. Clotting factor IX levels in C/EBP alpha knockout mice. Br J Haematol. 1997;99: 578-579.

53. Lee YH, Sauer B, Johnson PF, Gonzalez FJ. Dis ruption of the c/ebp alpha gene in adult mouse liver. Mol Cell Biol. 1997;17:6014-6022.

54. Choi BH, Park GT, Rho HM. Interaction of hepatitis $B$ viral $X$ protein and CCAAT/enhancer-binding protein alpha synergistically activates the hepatitis $B$ viral enhancer II/pregenomic promoter. J Biol Chem. 1999;274:2858-2865.

55. Garcia E, Lacasa M, Agli B, Giudicelli Y, Lacasa D. Modulation of rat preadipocyte adipose conversion by androgenic status: involvement of C/EBP transcription factors. J Endocrinol. 1999; 161:89-97. 